\title{
Terahertz electric response of fractal metamaterial structures
}

\author{
F. Miyamaru, ${ }^{1}$ Y. Saito, ${ }^{1}$ M. W. Takeda, ${ }^{1}$ B. Hou, ${ }^{2}$ L. Liu, ${ }^{2}$ W. Wen,${ }^{2}$ and P. Sheng ${ }^{2}$ \\ ${ }^{1}$ Department of Physics, Faculty of Science, Shinshu University, 3-1-1 Asahi, Matsumoto, Nagano 390-8621, Japan \\ ${ }^{2}$ Department of Physics, The Hong Kong University of Science and Technology, Clear Water Bay, Kowloon, Hong Kong
}

(Received 29 November 2007; published 18 January 2008)

\begin{abstract}
The electromagnetic response of $\mathrm{H}$-fractal structures, consisting of self-similar metallic lines or slits in metallic films deposited on silicon substrate, has been investigated in the terahertz region. The subwavelength fractal structure was observed to display electric resonances, with redshift in the resonant frequencies accompanying an increase in either the fractal level or the sample size. The associated increase in sample size is several times smaller than that expected from the scaling rule, indicating the possibility for achieving significantly subwavelength resonances. The very strong terahertz wave confinement in subwavelength regions (about 17 times smaller than the wavelength in vacuum), together with the multifrequency nature of the fractal resonances, characterize our metamaterial unit cell. By measuring both the power transmission and phase shift, we obtain the real and imaginary parts of the effective permittivity for our $\mathrm{H}$-fractal structures in the terahertz regime. Strongly dispersive behavior of the real part of the permittivity is seen at multiple frequencies.
\end{abstract}

DOI: 10.1103/PhysRevB.77.045124 PACS number(s): 42.70.Qs, 42.79.-e, 07.57.-c, 42.60.Da

\section{INTRODUCTION}

Photonic crystals have emerged as a class of artificial structures capable of manipulating electromagnetic waves, with potential for applications in many optical devices. ${ }^{1-4}$ Their unique characteristics arise from the periodic structure with periodicity comparable to the wavelength. Hence, in order to be effective, the overall size of a photonic crystal must be a few times the wavelength at least. In contrast, metamaterials are another class of wave-functional artificial structures which can manipulate waves with sample sizes that are substantially subwavelength. They have great potential to be the basis of novel terahertz component(s) unachievable with conventional materials. By modifying the permittivity and permeability with metamaterials, ${ }^{5}$ many exotic phenomena may be obtained, e.g., perfect lens with the negative refraction ${ }^{6}$ and the cloaking effect. ${ }^{7}$ Since the size of a metamaterial unit cell is generally subwavelength, the overall sample size can be smaller than that of photonic crystals. However, in the conventional study of metamaterials, the size of a unit cell is at most ten times smaller than the wavelength at operation frequency. For future metamaterial technology, e.g., near-field metamaterial devices, further downsizing of the metamaterial unit cell may be needed. Another drawback of the metamaterials is the narrow frequency range of operation, an issue which may also need to be addressed.

Subwavelength fractal structures have potential to make metamaterials with interesting resonant properties not achievable in conventional metamaterial components. In particular, owing to the unique fractal characteristic of diverging surface area within a finite volume (or diverging boundary length within a finite area), there is the possibility of fabricating metamaterials with sample sizes that are extremely small as compared to the wavelength at operational frequency. In addition, the fractal structure can also display multifrequency operation within a single unit, thus partially resolving the issue of operational frequency range.

Here, we demonstrate an approach for fabricating the terahertz metamaterials with multifrequency operation and tunability by using the $\mathrm{H}$-shaped fractal structure. The fractal structures, consisting of metallic lines (positive type of fractal structures) or slits in metallic films (negative type of fractal structures), exhibit electric resonances in the terahertz regime. Redshift of the resonant frequency was observed to accompany an increase in either the fractal level or the sample size. However, the increase in the sample size is many times smaller than that expected from the usual scaling rule. The ratio of fractal size to (the operational) wavelength decreases further with increasing fractal level, thus indicating the realistic possibility of making significantly more compact metamaterial devices with multifrequency operation.

\section{EXPERIMENT}

Fractal patterns are generated from a $40-\mu \mathrm{m}$-thick line of length $a_{1}=4000 \mu \mathrm{m}$, defined as the first level of the structure. The line of the second level $\left(a_{2}=4000 \mu \mathrm{m}\right)$ is oriented along the direction perpendicular to the first level and connected at its midpoint to the end of the first level line, forming part of an $\mathrm{H}$. The third and fourth levels are constructed similarly except the line length is scaled down by a factor of $2\left(a_{3}\right.$ and $\left.a_{4}=2000 \mu \mathrm{m}\right)$. By continuing this procedure, a self-similar $\mathrm{H}$-fractal structure is realized. The fractal dimension $D$ is defined as

$$
D=\frac{\log N}{\log (1 / S)}
$$

where $N$ is the number of new, smaller "H's," and $S$ is the reduction ratio of the new smaller H's (generator) to the initiator H. In our experiment, $N=4$ and $S=1 / 2$, so that the fractal dimension is $D=2$, meaning that our fractal structure covers the whole area of a square when the stage number approaches infinity. The occupied area of an $\mathrm{H}$ fractal increases with increasing fractal level, but it is at most a square with sides that are twice the length of the first-level line. Thus, ideally the length of the metallic line can diverge within a finite sample area. In practice, however, the maxi- 


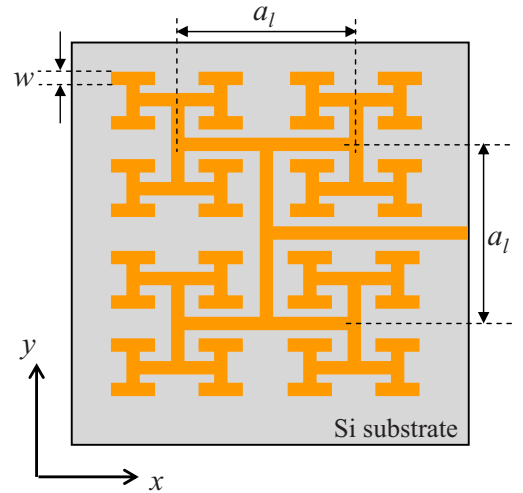

FIG. 1. (Color online) Schematic picture of an enlarged part of the fractal structure in the range from 7 th to 12 th level $(l)$. The fractal structure consists of $40 \mu \mathrm{m}$ wide $(w)$ metallic lines or slits with an $\mathrm{H}$-shaped mother element.

mum length is determined by the spatial resolution of the fabrication process.

Metallic fractal structures up to the 12th level $\left(a_{12}\right.$ $=125 \mu \mathrm{m}$, see Fig. 1) were evaporated on 510- $\mu \mathrm{m}$-thick, high resistivity silicon wafer. The transmission spectra were measured by the terahertz time-domain spectroscopy (TDS). ${ }^{8}$ Terahertz wave pulses were emitted from a bowtie-type photoconductive antenna illuminated by a $120 \mathrm{fs}$ laser pump pulses at $800 \mathrm{~nm}$. The emitted terahertz wave pulses were collimated with an off-axis paraboloidal mirror to a diameter of $\sim 8 \mathrm{~mm}$, and normally incident on the fractal structure. Transmitted terahertz wave was detected by another bowtietype photoconductive antenna gated with time-delayed probe laser pulses, diverted from the pump pulses. The incident terahertz wave was linearly polarized either parallel or perpendicular to the first-level metallic line of the fractal. By Fourier transform, the measured wave forms of the terahertz wave was converted into spectra of power transmittance and phase shifts, allowing direct estimation of complex permittivity $^{8}$ of the metamaterial when the permeability is assumed to be constant. In our experiment, the magnetic field of the incident terahertz wave was along the surface; hence, there can be no magnetic response in the measured data.

\section{TRANSMISSION CHARACTERISTICS}

\section{A. Positive type fractal structure}

We first investigate the transmission response of the positive type fractals. Figure 2(a) shows the power transmittance of the fractal metamaterial (with 11 levels) as a function of frequency at normal incidence. Black squares denote the spectrum for the incident electric field polarized parallel to the $y$ axis defined in Fig. 1, while red circles denote the case where the electric field was polarized parallel to the $x$ axis. For the incident electric field parallel to the $y$ axis, a sharp stop band is observed at $0.095 \mathrm{THz}(\mathrm{SB} 1)$. When the incident electric field was rotated by $90^{\circ}$, two stop bands are seen at 0.052 (SB2) and $0.173 \mathrm{THz}$ (SB3), showing a clear dependence on the incident azimuthal polarization. The theoretical results calculated by the finite difference time do-

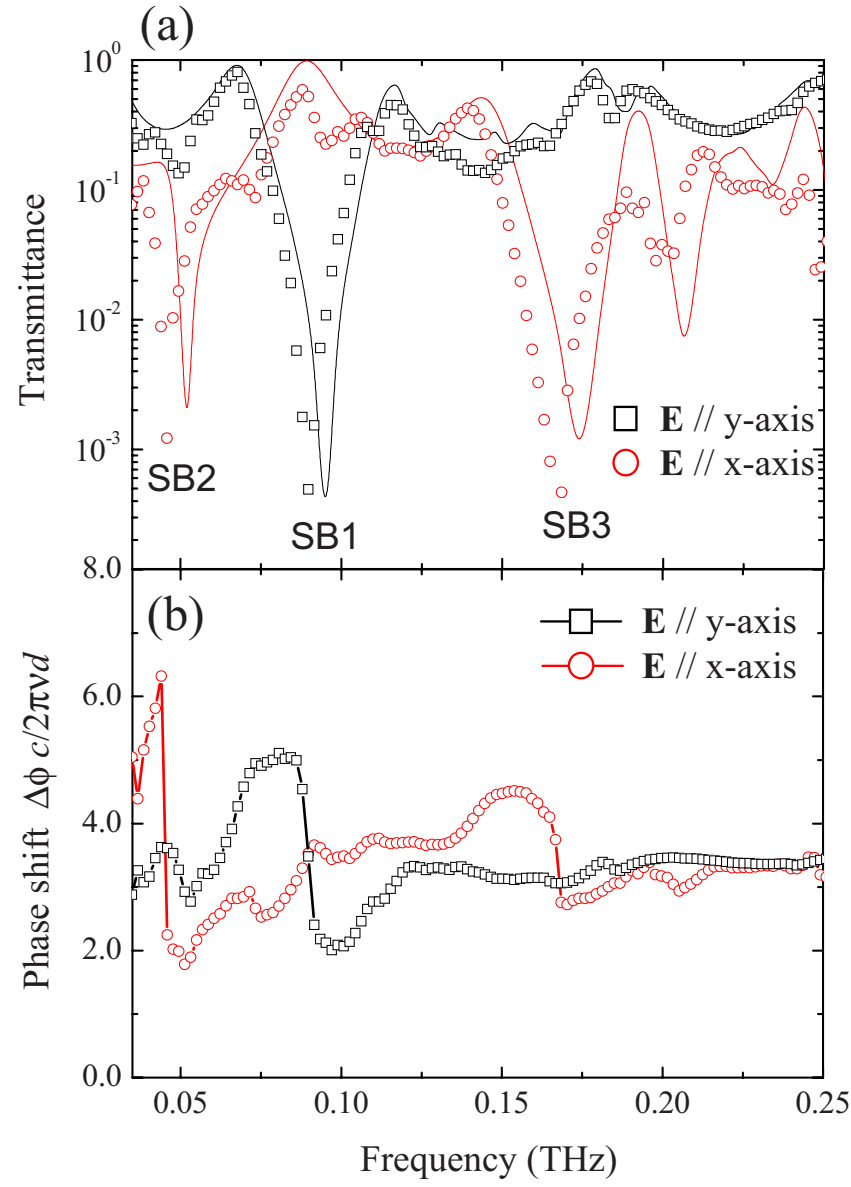

FIG. 2. (Color online) (a) Measured (markers) and calculated (lines) transmission spectra and (b) measured phase spectra of a 11-level fractal structure for the incident polarization parallel (black square) and perpendicular (red circle) to the $y$ axis, as depicted in Fig. 1. The phase spectra is normalized by $2 \pi d \nu / c$, where $\nu$ is the frequency, $d$ the thickness of the sample, and $c$ the speed of light.

main method are also shown as solid curves in Fig. 2(a) for each of the two polarizations. Good agreement between experiment and theory is seen. The slight discrepancy is due to the imperfection of the fractal structure and the permittivity of the $\mathrm{Si}$ substrate assumed in the calculation. These stop bands that owe their origin to localized resonances are characteristic of the fractal structure. ${ }^{9}$ The stop band SB1, for example, with the electric field localized around the lines of the ninth level, is indicative of the electric dipole resonance along the metallic line. The wavelength in vacuum at resonant frequency $\left(\lambda_{\mathrm{SB} 1}=3160 \mu \mathrm{m}\right)$ is 6.3 times longer than that expected from the half wavelength resonance of the simple dipole model with the same line length $\left(\lambda_{\text {dipole }} / 2\right.$ $=250 \mu \mathrm{m}) .{ }^{10}$

Since we would like to use terahertz-TDS spectroscopy to determine the characteristics of the fractals, it is necessary to measure the phase information of the transmitted terahertz wave as well. Figure 2(b) shows the measured phase shift spectra for incident polarizations parallel to the $y$ (black square line) and $x$ (red circle line) axes. Here, the original phase shift $\Delta \phi$ is normalized by $2 \pi d \nu / c$, with $\nu$ being the frequency, $d$ the thickness, and $c$ the speed of light in 


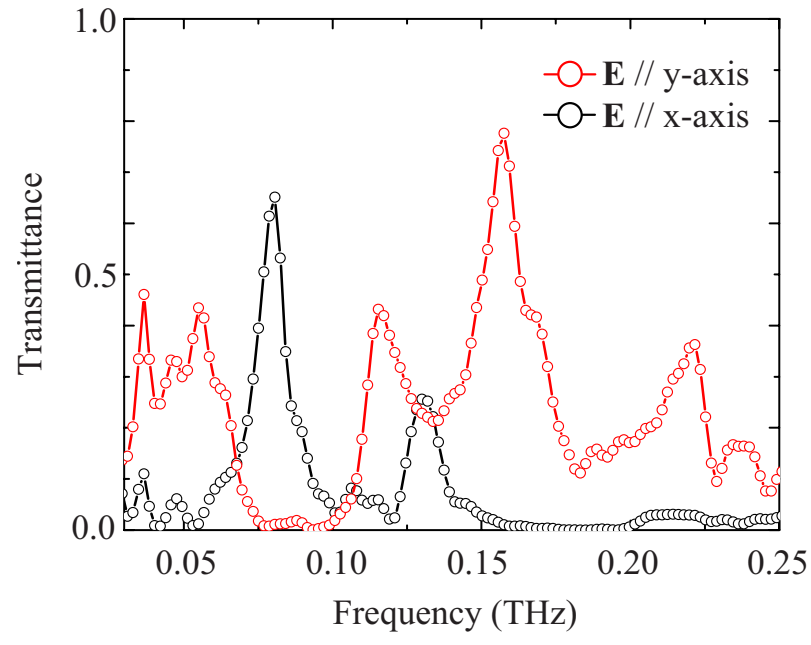

FIG. 3. (Color online) Measured transmission spectra of a 11level negative-type fractal structure for incident polarization parallel (red circle) and perpendicular (black circle) to the $y$ axis.

vacuum. Dispersive behavior is seen at the same frequencies of the transmission stop bands, implying an alteration of the effective permittivity value from that of the constituent material. This is further discussed in Sec. IV.

\section{B. Negative type fractal structure}

The transmission spectra of the complementary type (negative type) of fractal structures were also investigated. Figure 3 shows the zeroth order transmission spectra for negative type fractals, the geometrical parameters of which are same to those of the positive type of fractal shown in Fig. 2. The resonant transmission peaks were observed for both incident polarizations parallel (solid line) and perpendicular (dashed line) to the $y$ axis. The observed peak frequencies, with the incident polarizations rotated 90 degrees, are located at the same frequencies as those of transmission dips observed in Fig. 1(a) for the positive fractal. This is a manifestation of Babinet's principle for complementary structures. These resonant peaks are attributable to localized resonances at corresponding fractal levels, and are the same as those of positive type fractals. In this case, the energy of the terahertz wave at resonant frequency is strongly confined in the subwavelength slits. Such subwavelength behavior will be further discussed in Sec. VI.

\section{ELECTROMAGNETIC CHARACTERISTICS OF H- FRACTAL METAMATERIALS}

In this section, we investigate the electromagnetic characteristics of $\mathrm{H}$ fractals as metamaterials. In general, the effective permittivity and permeability are controlled by the structure of metamaterials. In our experiment, since the measurements are performed at normal incidence, the electric and magnetic fields both lie in the fractal metamaterial plane and thus we can consider only the effective permittivity. The bianisotropic effect, ${ }^{11}$ in which the external electric field couples to the magnetic response, is excluded from our discussion because of the symmetry of the fractal structure.

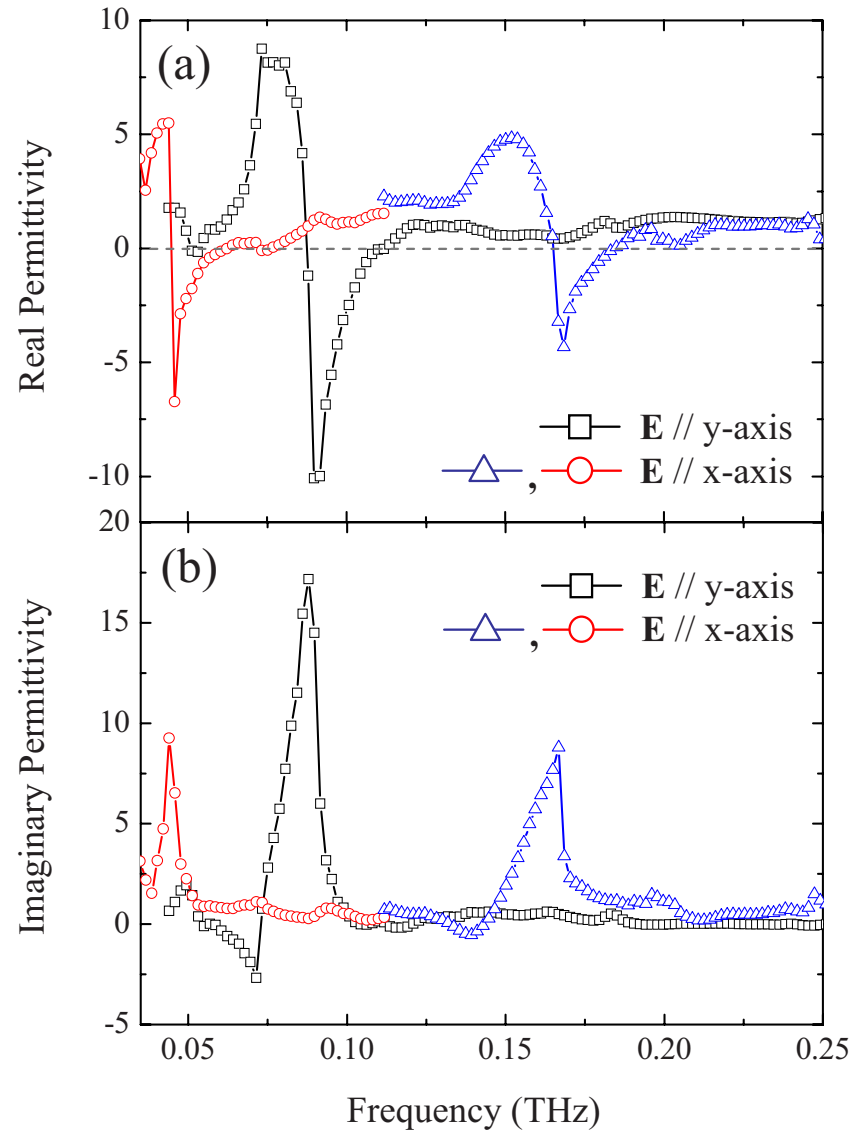

FIG. 4. (Color online) (a) Real and (b) imaginary parts of the effective permittivity for the fractal metamaterial. Black square is for the incident polarization parallel to the $y$ axis and blue triangle and red circle are for the polarization perpendicular to the $y$ axis.

Figures 4(a) and 4(b) show the real and imaginary parts of the effective complex permittivity, $\widetilde{\varepsilon}=\varepsilon_{1}+i \varepsilon_{2}$, respectively, estimated from the transmission and phase shift spectra. We assumed the effective thickness $d_{\text {eff }}$ of the metamaterial to be equal to the periodicity ${ }^{12,13}$ of the relevant level of the resonance, i.e., $d_{\mathrm{eff}}=500 \mu \mathrm{m}$ for SB1. In this estimation, the contribution of the Si substrate is subtracted except the red shift of the resonant frequency from that for the freestanding fractal metamaterial due to the refractive index of $\mathrm{Si}\left(n_{\mathrm{Si}} \approx 3.4\right.$ at $0.1 \mathrm{THz})$. For the incident polarization parallel to the $y$ axis, strong dispersive behavior of $\varepsilon_{1}$ is seen at around $0.095 \mathrm{THz}$ where a peak is observed in $\varepsilon_{2}$. The magnitude of $\varepsilon_{1}$ is observed to vary from 8.5 down to -10 . Negative values of $\varepsilon_{2}$ are observed at around $0.7 \mathrm{THz}$. These unexpected values are artifacts as the result of the imperfect compensation of the $\mathrm{Si}$ substrate effect. Owing to the high permittivity of $\mathrm{Si}$ $\left(\varepsilon_{1}=11.3\right.$ at $\left.0.1 \mathrm{THz}\right)$, the strong Fabry-Pérot fringes appear in the transmission spectrum, thus misleading the estimation of the transmittance of the fractal metamaterial. For the incident polarization parallel to the $x$ axis, similar dispersive characteristics in $\varepsilon_{1}$ are observed at around the other two frequencies, corresponding to the resonant frequencies of SB2 and SB3. This indicates the broadband modulation of the complex permittivity to be achievable by using the fractal structure. 
It should be noted that the concept of effective permittivity or permeability is based on the requirement that the relevant size of the resonant structure should be small compared to the operational wavelength. However, different resonances have different resonant structure sizes and operational wavelengths. Thus, some attention is needed for defining the "unit cell" of each resonance for estimating the effective permittivity. For different resonant frequencies, the effective medium description may be applied up to that high resonant frequency at which diffraction occurs, i.e., when the unit cell size corresponding to the lower resonant frequency matches the wavelength of that high resonant frequency. When that occurs, the localized resonances at the higher resonant frequencies would couple to the "Bloch scatterings," and "hybrid" modes can occur. The effective medium treatment would become questionable at that point.

\section{DEPENDENCIES ON STRUCTURAL PARAMETERS}

\section{A. Dependence on fractal level}

To further investigate the terahertz response of fractal structures, we have measured the transmission spectra with different fractal levels. Figure 5(a) shows the transmission spectra for three fractals with 10 (red solid line), 11 (black dotted line), and 12 (blue dashed line) levels. The incident terahertz wave was polarized parallel to the $x$ axis. The resonant frequency located at $0.053 \mathrm{THz}$ for the ten-level fractal is seen to shift to lower frequencies with increasing fractal level, reaching $0.037 \mathrm{THz}$ for the 12-level structure. A similar redshift of the resonant frequency is observed for the higher stop band, varying from 0.184 to $0.117 \mathrm{THz}$. This redshift with increasing fractal level offers an advantage for making very small, subwavelength resonant structures. In Fig. 5(b), we plot the size of the resonant structure as a function of the operational wavelength. The size $\left(S_{\text {norm }}\right)$ and operational wavelength $\left(\lambda_{\text {norm }}\right)$ are normalized by those of the ten-level fractal. In general, the area of the planar resonant structure follows the $\lambda_{\text {norm }}^{2}$ scaling rule, shown as the dotted line in Fig. 5(b). The necessary area for the resonant fractal structures in our experiment, however, is smaller than that predicted by the conventional scaling rule, and the difference increases with wavelength. This property is peculiar to the fractal structures, and it helps in the reduction of unit cell size of resonant structures.

The origin of the resonant frequency tuning is the extension of the effective length for the terahertz wave. With increasing fractal level, the capacitance and inductance are effectively appended at both ends of the metallic line. These additional capacitance and inductance make the effective electric length longer. This is analogous to shortening of the physical length in the capacitance and inductance loaded dipole antenna. Especially in the present $\mathrm{H}$-fractal structure with $D=2$, we can elongate the effective length of the metallic line to infinity in the ideal case. Therefore, the extremely small size of resonant structure with respect to the operational wavelength is a realizable possibility.

\section{B. Dependence on linewidth}

As shown in the previous section, the resonant frequencies show a redshift with increasing fractal level. This indi- (a)
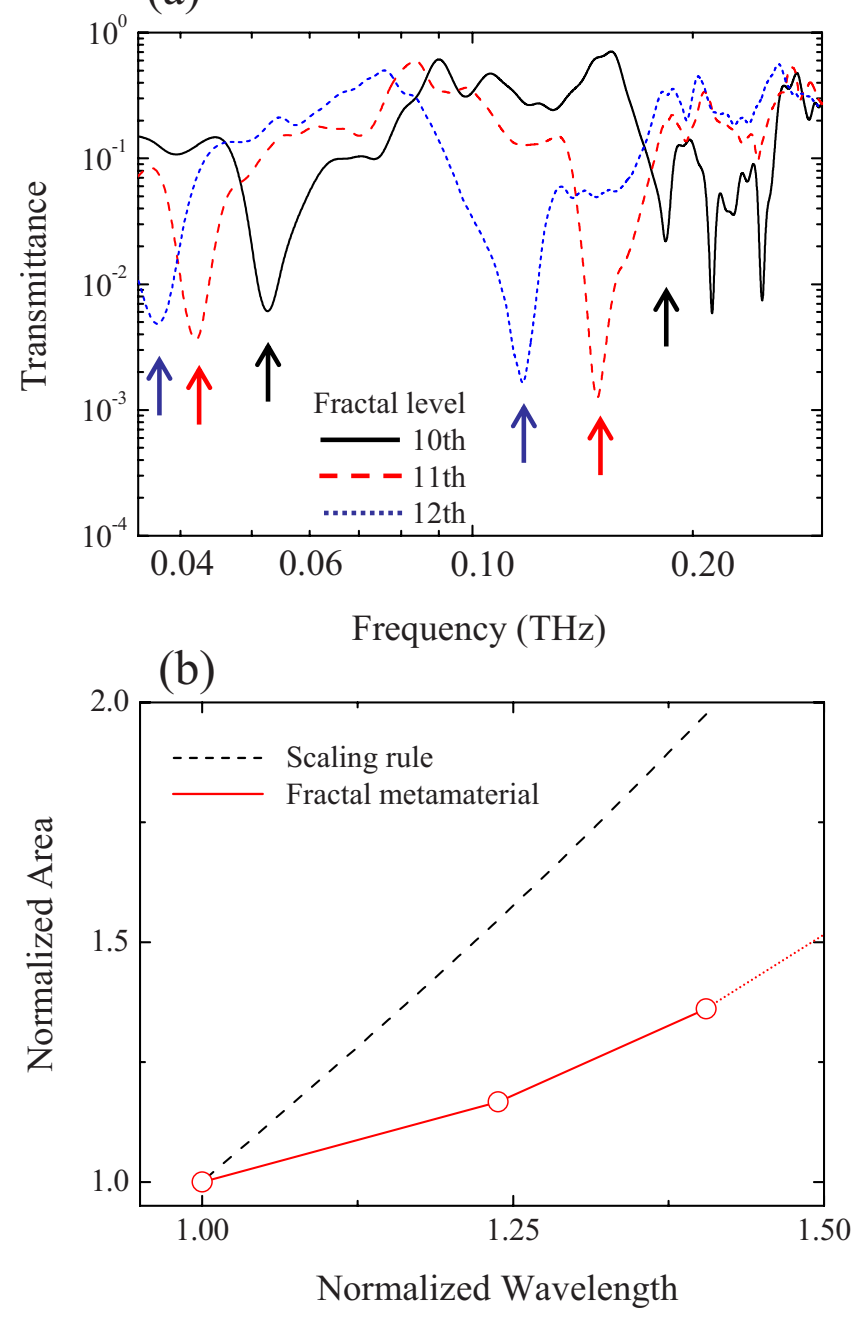

FIG. 5. (Color online) (a) Dependence of the transmission spectra on the fractal level. Red shift of the resonant frequencies (of stop bands) is seen with increasing the fractal level. (b) Comparison of the required metamaterial area for the fractal metamaterial and the conventional scaling rule.

cates that the geometrical size of the fractal with respect to the resonant wavelength can become smaller with increasing fractal level. For the $\mathrm{H}$ fractal, even if the fractal level goes to infinity, the area of the fractal can reach at most four times as large as that of the initiator $\mathrm{H}$, yet the resonant wavelength has the potential to become extremely large. In practice, however, since the width of metallic line is finite, the feasible maximum fractal level has to be bounded. The thinner metallic linewidth, the larger the maximum achievable fractal level. Based on this perspective, the dependence of the transmission characteristic on metallic linewidth is critically important in practical fabrication. Figures 6(a)-6(c) show the transmission spectra of positive type of fractals, the linewidths of which are 54, 40, and $25 \mu \mathrm{m}$, respectively. The fractal level of these samples is 11 . The resonant transmission stop bands are observed at similar frequencies for all samples. This result indicates that even by using the narrow metallic line of $24 \mu \mathrm{m}$, similar resonance frequency with that of wider linewidth can be achieved. Hence, using higher 


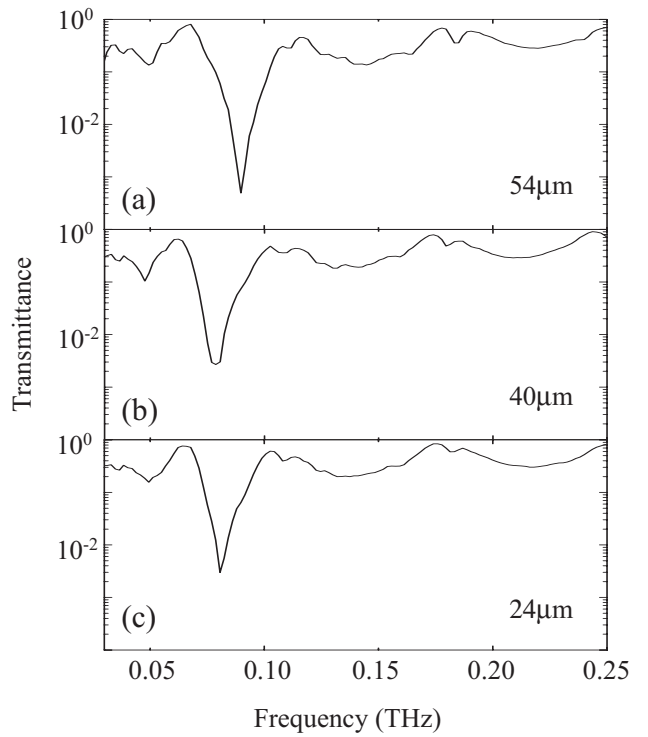

FIG. 6. Measured transmission spectra of 11-level positive type fractals with linewidths of (a) $54 \mu \mathrm{m}$, (b) $40 \mu \mathrm{m}$, and (c) $25 \mu \mathrm{m}$.

fractal level to lower the resonance frequency is a realistic and viable option.

\section{SUBWAVELENGTH CONFINEMENT OF TERAHERTZ WAVE FIELD}

For the negative type fractal structure, the subwavelength characteristics are manifested in the terahertz wave confinement. Figure 7(a) shows the calculated transmission spectra of the negative type fractal structures of 10 (red solid line), 11 (black dotted line), and 12 (blue dashed line) levels, the geometrical parameters of which are the same as that of the metallic line structure used in the experiment shown in Fig. 3. In the transmission spectrum of the ten-level fractal, a high resonant peak with more than $85 \%$ transmittance is seen at $0.107 \mathrm{THz}$. The resonant peak frequency shows a clear redshift with increasing fractal level similar to that of the stop bands for the positive type fractal structures [Fig. 5(a)]. Figures 7(b)-7(d) show the electric field distribution in the ranges from 7 th to 12th, 11th, and 10th levels for the fractal structures with 12, 11, and 10 levels, respectively, at each resonant frequency. In Fig. 7(d), the electric field is localized in the slit of the 9th level, in which the long axis is about $250 \mu \mathrm{m}, \sim 1 / 12$ the wavelength at $0.107 \mathrm{THz}$. Even if we take into account the shortening of the wavelength in the $\mathrm{Si}$ substrate $\left(n_{\mathrm{Si}}=3.4\right)$, the length of the long axis is still $1 / 3.5$ the wavelength in $\mathrm{Si}$. This subwavelength localization of the terahertz wave in the fractal structure was also observed in other frequencies. ${ }^{13}$ Interestingly, we find the confinement size to be almost invariant with increasing fractal level, while the resonant frequency becomes lower. Only a slight penetration into the next fractal level is observed in Fig. 7(b). This result implies that significantly subwavelength spot can be realized with the fractal structure. Indeed, for the 12-level fractal structure, the confinement size is about $1 / 17$ that of the relevant wavelength. As discussed above, since the additional higher fractal levels provide the loaded capacitance

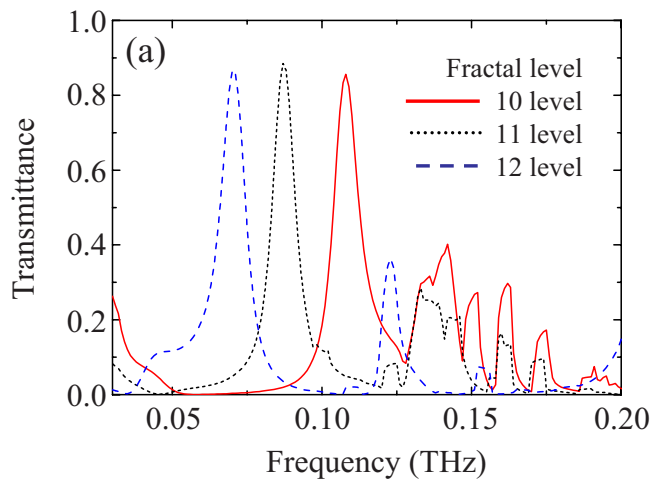

(b) (c) (d)

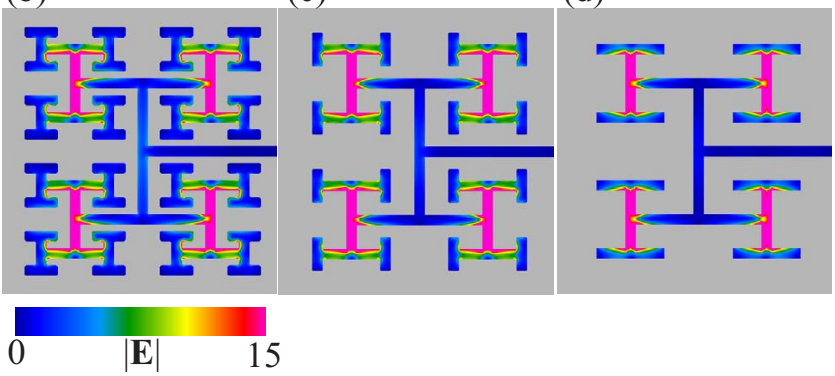

FIG. 7. (Color online) (a) Simulated transmission spectra of a metallic slit fractal structure for 10 (black solid line), 11 (red dashed line), and 12 (blue dotted line) levels. [(b)-(d)] Simulated electric field distributions in the 7th to 12th, 11th, and 10th levels for each of the three fractal structures, respectively. The $|E|$ values shown are normalized with respect to the incident electric field.

and inductance, the resonant wavelength can be longer without extending the physical size of the sample, leading to the subwavelength confinement of the terahertz wave.

It should be noted that although the air fraction of the fractal structure is at most $40 \%$, the transmittance at the resonant peak exceeds that, owing to the resonant phenomenon. Since the transmitted terahertz wave at resonant frequency is limited only in the slit of the relevant fractal level, the spatial distribution of the terahertz wave is rearranged into a further smaller area. At the peak frequency of $0.070 \mathrm{THz}$ of the 12 -level fractal, for example, the power of $85 \%$ transmittance is focused into an area which is only $2.8 \%$ of the whole sample area. This very significant confinement of the terahertz wave field can be valuable for the sensing or imaging technology of near-field optics, where subwavelength light confinement is critically important. The electric field localization in the gap of the metallic structure has been observed in other structures used as unit cells of metamaterials. ${ }^{14}$ However, if the positive type of metallic fractal structure is used, such localization of the electric field is relatively leaky, and the ratio of the localized field with respect to the whole incident field might be small. Thus, strong electromagnetic field confinement might not be expected for the positive type of fractal structures. The negative-type metallic fractal structure (i.e., slits in metal films), however, has the potential to strongly confine the electromagnetic field in the very small area. Here, the fractal structure is crucial, since simple circular or rectangular holes in metallic films are known to have a cutoff wavelength. For rectangular holes, the cutoff wavelength is twice the width of holes; hence, efficient subwave- 
length wave field confinement cannot be expected. For the negative-type fractals investigated in this paper, however, the terahertz wave field can be confined in the subwavelength region with relatively high transmission efficiency. This is due to the very slight spreading of the localized terahertz wave field in the slits of the negative type fractals, but further detailed study is needed to make clear the near field behavior.

\section{CONCLUDING REMARKS}

In summary, we have investigated the electric resonant characteristics of the $\mathrm{H}$ fractals in the terahertz regime. We find the resonant frequency to exhibit a redshift with increasing fractal level, where the increase in the overall area of the fractal is much smaller than that expected from the scaling rule. This indicates the possibility of making compact metamaterial devices, with multifrequency operation, by utilizing fractal structures. Such subwavelength compact metamaterial devices may be useful for high resolution nearfield optics.

\section{ACKNOWLEDGMENTS}

F.M., Y.S., and M.W.T. wish to acknowledge the support by the Grant-in-Aid for Scientific Research (S) No. 171067010 under the auspice of the Ministry of Education, Culture, Sports, Science and Technology, Japan. B.H., L.L., W.W., and P.S. gratefully acknowledge the support of Hong Kong RGC under Grant No. CA06/07.SC04.
${ }^{1}$ E. Yablonovith and K. M. Leung, Nature (London) 351, 278 (1991).

${ }^{2}$ S. Noda, K. Tomoda, Y. Noritsugu, and A. Chutinan, Science 289, 604 (2000).

${ }^{3}$ S.-Yu. Lin, E. Chow, V. Hietala, P. R. Villeneuve, and J. D. Joannopoulos, Science 282, 274 (1998).

${ }^{4}$ F. Miyamaru and M. Hangyo, Phys. Rev. B 71, 165408 (2005).

${ }^{5}$ J. B. Pendry, A. J. Holden, D. J. Robbins, and W. J. Stewart, IEEE Trans. Microwave Theory Tech. 47, 2057 (1999).

${ }^{6}$ J. B. Pendry, Phys. Rev. Lett. 85, 3966 (2000).

${ }^{7}$ J. B. Pendry, D. Schurig, and D. R. Smith, Science 312, 1780 (2006).

${ }^{8}$ M. Hangyo, T. Nagashima, and S. Nashima, Meas. Sci. Technol.
13, 1727 (2003).

${ }^{9}$ W. Wen, L. Zhou, J. Li, W. Ge, C. T. Chan, and P. Sheng, Phys. Rev. Lett. 89, 223901 (2002).

${ }^{10}$ W. L. Stutzman and G. A. Thiele, Antenna Theory and Design (Wiley, New York, 1998).

${ }^{11}$ N. Katsarakis, T. Koschny, M. Kafesaki, E. N. Economou, and C. M. Soukoulis, Appl. Phys. Lett. 84, 2943 (2004).

${ }^{12}$ B. I. Popa and S. A. Cummer, Phys. Rev. B 72, 165102 (2005).

${ }^{13}$ W. Wen, L. Zhou, B. Hou, C. T. Chan, and P. Sheng, Phys. Rev. B 72, 153406 (2005).

${ }^{14}$ H.-T. Chen, W. J. Padilla, J. M. O. Zide, A. C. Gossard, A. J. Taylor, and R. D. Averitt, Nature (London) 444, 597 (2006). 